\title{
Non-dimensional Characteristics of Open Wet Clutches for advanced Drag Torque and Aeration Predictions
}

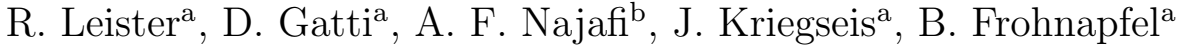 \\ ${ }^{a}$ Institute of Fluid Mechanics, Karlsruhe Institute of Technology (KIT), Kaiserstraße 10, \\ 76131 Karlsruhe, Germany \\ ${ }^{b}$ School of Mechanical Engineering, College of Engineering, University of Tehran P.O. \\ Box 11365/4563, Tehran, Iran
}

\begin{abstract}
The remaining torque of disengaged wet clutches is a major source of energy loss and, therefore, an objective of current research. The present contribution describes the necessary simplifications to obtain an analytical solution of the governing equations by means of an order-of-magnitude analysis. The obtained results are brought to a dimensionless frame of reference, where formerly unknown simple dependencies of the drag torque and the aeration onset have been uncovered. The dimensionless description serves as promising a means to achieve a quantitative comparison of experimental data. Additionally, a new modelling concept for grooves is introduced, which is based on the hydraulic-diameter concept. The combination of either approach offers a robust prediction method for drag torque and aeration onset.
\end{abstract}

Keywords: Open clutch flows, Dimensional analysis, Analytical solution, Modelling concepts

\section{Introduction}

Energy-saving trends remain highly important in automotive industry due to a strict regulation of vehicle $\mathrm{CO} 2-$ emisson and the potential of a higher range for battery-powered vehicles. A current field of optimization 5 are wet clutches, which are part of nearly every higher-class automobile. The remaining torque in disengaged state is a major source of energy losses and consequently an essential research topic in this area with the aim to predict and minimize these losses. The flow scenario in open wet clutches can be classified as a rotor-stator configuration with through flow. Figure 1 shows 
the simplified geometry of an open wet clutch model with only one rotor and one stator disk. Important parameters are the volumetric flow rate $Q$, which is centrally supplied to counter-act heat generation during the engagement process, the gap height $h$ between both disks, which adjusts autonomously in wet clutches, the inner and outer radius of the rotating disk $R_{1}$ and $R_{2}$, the angular velocity $\Omega$ and the drag torque $T_{s}$, which is caused by the speed difference of driving and output unit and thus the shearing of the oil.

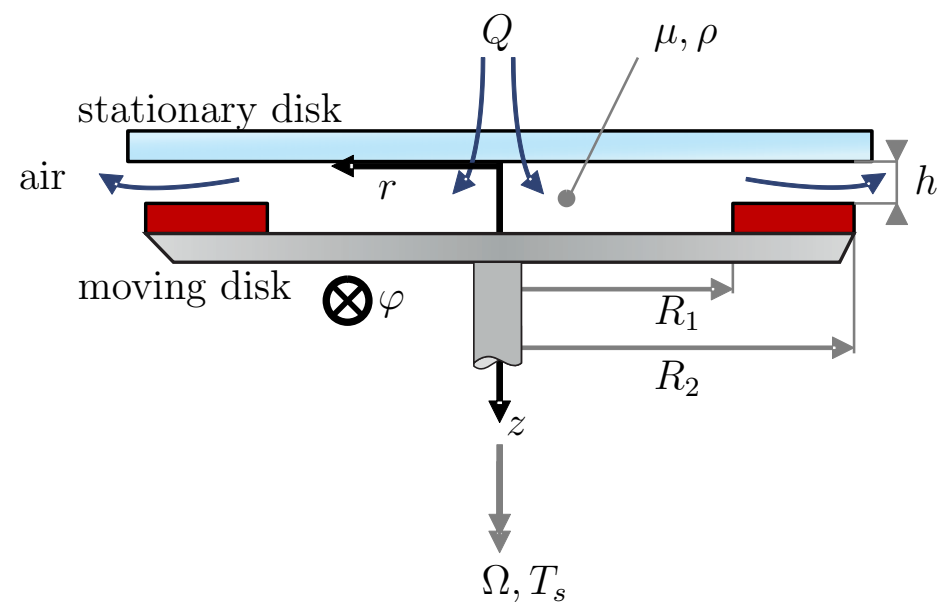

Figure 1: Sketch of an open clutch with all geometry and operation parameters, and fluid properties.

At low differential speed the gap is completely filled with oil, such that all oil passes through the gap and accordingly no two-phase flow occurs. Increasing the rotational speed of the moving disk results in a likewise increased tangential momentum of the oil and consequently in a higher centrifugal force component. At a certain point the conveying capacity exceeds the constant volumetric flow rate $Q$, which leads to air induction from the surrounding into the gap at the outer radius $R_{2}$. With higher speed difference the presence of air in the gap leads to a significant reduction of drag torque, since viscosity of air is much lower than the one of the cooling oil.

Knowledge of the onset of this so-called aeration is extremely desired information for the design of clutches, since this onset in turn leads to a subsequent drop of drag torque due to the presence of air in the rotor-stator gap. Accordingly, the major objective of theoretical clutch-flow modelling efforts revolves around an accurate prediction of this onset on the basis of known 
(geometrical and operational) parameters of an operative open wet clutch. The attempt of a theoretical description of the drag torque was strongly driven by automotive companies, where the potential of drag reductions was first discovered and described (e.g. [1]). Different configurations of surface structures on the disk have proven to result in a reduction of this maximum drag torque in open clutch flows. These surface modifications shift the onset of aeration to lower rotational speeds, thus reducing the maximum drag torque of the system. However, the two-phase flow still hasn't been understood in detail, due to restrictions of the measurement techniques on one side and limitations of the numerical simulations on the other side.

Over the last years the topic of open clutch flows has been studied with numerical, analytical and experimental approaches. One of the first suggestions was made by KATO et al. [2] who modified an approach of HAsHimoto et al. [3], which originally was used for thrust bearings. This approach assumed a turbulent flow and calculated the drag torque with the help of an empirical formulation. YUAN et al. [4] used this approach to propose a model in consideration of an introduced shrinking of the oil film and a classification of the flow by use of dimensionless numbers. A turbulent flow in the gap seems however unlikely, due to a small gap ratio $G=h / R_{2}$ in the order of $10^{-3}$ and a moderate Reynolds number (see LAUNDER et al. [5] for more details on the classification of rotor-stator flows).

KitabayAshi et al. [6] assumed a laminar flow and used a laminar shear stress model to describe the drag torque characteristics in the single-phase region. While this model did not consider the onset of aeration, a new group of laminar models attempted to overcome this shortcoming. The basis of all these models is a laminar shear stress approach given as

$$
\tau_{\varphi z}=\mu \frac{\partial u_{\varphi}}{\partial z}
$$

RAO [7] combined the shrinking of the oil film of [4] and the laminar approach of [6] and formed a laminar shear stress model, which is capable to predict the decrease of the drag torque in the two-phase region and provides a precise description of the laminar single-phase flow. Several new models with slightly different terms were published in the following years. HuANG et al. [8] modelled a hydro-viscous drive with this concept and analysed the velocity profiles in the single-phase region. The model of IQBAL et al. [9, 10] considered the fluid and the mist contributing to the resulting torque. The most recent models and experimental validation were conducted by PAHLOVY 
et al. [11, 12, 13], where surface tension and high rotational speed differences were considered.

Due to the complex nature of two-phase flow, these models rely on a simplified description as basis for drag torque calculations, i.e. the concept though this concept is in good agreement with the measurements of the drag torque, the fluid mechanic conditions in the lubrication gap are not taken into account.

The development of an analytical model was accompanied by various atThe respective experimental studies however focused mainly on the generation of validation data for the analytical models. More recently, a systematic experimental investigation and a profound comparison of analytical models has been provided by NEUPERT et al. [14.

In continuation of these efforts [14, the present paper provides a detailed discussion of the simplification of the governing equations of the flow between the disks required as to obtain the analytical prediction for torque and aeration onset found in literature. Particular emphasis is placed on a correct derivation of the involved interrelationships from a fluid mechanic point of view, where it will be demonstrated that a transfer of the above solution for ungrooved disks to dimensionless numbers allows a direct and straight forward prediction of torque and aeration onset for different clutch geometries and operating conditions without consideration of further equations. Accordingly, a dimensionless diagram is introduced, in which torque mulation is proposed and tested on existing experimental results, which also comprises the formerly missing information on surface groove dimensions as influential quantities for the aeration onset.

\section{Dimensional analysis and aeration model}

Independent from any specific aeration or torque model the drag torque for open wet clutch flows depends on the interplay of seven other dimensional parameters (cp. Figure 1). Thus the desired quantity $T_{s}$ can be written as

$$
T_{s}=f\left(h, R_{1}, R_{2}, \nu, \rho, \Omega, Q\right),
$$

95

where in addition to the above-introduced quantities the density $\rho$ and kinematic viscosity $\nu$ of the oil at hand appear in Eq. (2). 
Dimensional analysis according to e.g. YARIN [15] reduces these interdependencies to the five dimensionless parameters

$$
\begin{gathered}
G=\frac{h}{R_{2}} \quad \text { gap ratio, } \\
\beta=\frac{R_{1}}{R_{2}} \quad \text { radii ratio, } \\
R e_{l}=\frac{R_{2} h \Omega}{\nu} \quad \text { lubrication Reynolds number, } \\
Q^{*}=\frac{Q \nu}{\Omega^{2} R_{2}{ }^{2} h^{3}} \quad \text { non-dimensional volume flow rate, } \\
\zeta_{m}=\frac{T_{s}}{\frac{\rho}{2} R_{2}{ }^{5} \Omega^{2}\left(1-\beta^{4}\right)} \quad \text { moment coefficient. }
\end{gathered}
$$

Note that $\zeta_{m}$ in the present context is a non-dimensional drag torque measure, similar to the moment coefficient defined in e.g. [16] or [17]. $G$ is the gap ratio, which is typically in the order of $10^{-3}-10^{-2}$ for open clutch flows. The second geometrical parameter $\beta$ is important for any drag torque calculation, due to the fact, that a significant amount of torque is generated only between $R_{1}$ and $R_{2}$. The so-called lubrication Reynolds number $R e_{l}$ is based on the circumferential velocity $\Omega R_{2}$ and the gap height, which is a classical formulation in tribology [18, 19]. This Reynolds number fits well to the flow scenario of open clutch flows as shown later. The last parameter is the nondimensional volume flow rate $Q^{*}$. It is worth to furthermore mention that the kinematic and dynamic viscosities are coupled via $\mu=\rho \nu$. The dimensionless quantities (3) are used below to simplify and in turn generalize the results of the torque and aeration model, which is derived in the following sections.

\subsection{Governing equations, assumptions and simplifications}

In cylindrical coordinates the conservation of mass for an incompressible flow reads

$$
\frac{1}{r} \frac{\partial}{\partial r}\left(r u_{r}\right)+\frac{1}{r} \frac{\partial u_{\varphi}}{\partial \varphi}+\frac{\partial u_{z}}{\partial z}=0 .
$$

The corresponding momentum conservation for the steady state flow of a Newtonian fluid is given by

$$
\rho\left(\frac{\partial u_{r}}{\partial t}+u_{r} \frac{\partial u_{r}}{\partial r}+\frac{u_{\varphi}}{r} \frac{\partial u_{r}}{\partial \varphi}-\frac{u_{\varphi}^{2}}{r}+u_{z} \frac{\partial u_{r}}{\partial z}\right)=
$$




$$
\begin{gathered}
-\frac{\partial p}{\partial r}+\mu\left\{\frac{\partial}{\partial r}\left[\frac{1}{r} \frac{\partial}{\partial r}\left(r u_{r}\right)\right]+\frac{1}{r^{2}} \frac{\partial^{2} u_{r}}{\partial \varphi^{2}}-\frac{2}{r^{2}} \frac{\partial u_{\varphi}}{\partial \varphi}+\frac{\partial^{2} u_{r}}{\partial z^{2}}\right\}+\rho g_{r} \\
\rho\left(\frac{\partial u_{\varphi}}{\partial t}+u_{r} \frac{\partial u_{\varphi}}{\partial r}+\frac{u_{\varphi}}{r} \frac{\partial u_{\varphi}}{\partial \varphi}+\frac{u_{r} u_{\varphi}}{r}+u_{z} \frac{\partial u_{\varphi}}{\partial z}\right)= \\
-\frac{1}{r} \frac{\partial p}{\partial \varphi}+\mu\left\{\frac{\partial}{\partial r}\left[\frac{1}{r} \frac{\partial}{\partial r}\left(r u_{\varphi}\right)\right]+\frac{1}{r^{2}} \frac{\partial^{2} u_{\varphi}}{\partial \varphi^{2}}+\frac{2}{r^{2}} \frac{\partial u_{r}}{\partial \varphi}+\frac{\partial^{2} u_{\varphi}}{\partial z^{2}}\right\}+\rho g_{\varphi} \\
\rho\left(\frac{\partial u_{z}}{\partial t}+u_{r} \frac{\partial u_{z}}{\partial r}+\frac{u_{\varphi}}{r} \frac{\partial u_{z}}{\partial \varphi}+u_{z} \frac{\partial u_{z}}{\partial z}\right)= \\
-\frac{\partial p}{\partial z}+\mu\left[\frac{1}{r} \frac{\partial}{\partial r}\left(r \frac{\partial u_{z}}{\partial r}\right)+\frac{1}{r^{2}} \frac{\partial^{2} u_{z}}{\partial \varphi^{2}}+\frac{\partial^{2} u_{z}}{\partial z^{2}}\right]+\rho g_{z}
\end{gathered}
$$

in radial $r$, circumferential $\varphi$ and axial directions $z$, respectively. Accordingly, $u_{r}, u_{\varphi}$ and $u_{z}$ are the corresponding velocity components. Together with the no-slip and impermeability boundary conditions on the solid walls these Navier-Stokes equations describe the flow field in the gap of an open wet clutch.

In order to obtain an analytical solution for the given flow field of an open wet clutch a number of assumptions are common for all existing models [7, 8, 9, 11, 20]. In addition to the aforementioned consideration of a steady and incompressible flow of a Newtonian fluid these assumptions are:

(i) laminar flow

(ii) no influence of gravity on the flow field

(iii) symmetric flow around z-axis

The commonly considered simplification of negligibly small axial flow component $u_{z}$ is not applied a priori in the present work as will be elaborated in the following.

Consideration of assumption (i)-(iii) reduces the governing equations (4) (7) to

$$
\begin{gathered}
\frac{1}{r} \frac{\partial\left(r u_{r}\right)}{\partial r}+\frac{\partial u_{z}}{\partial z}=0 \\
u_{r} \frac{\partial u_{r}}{\partial r}-\frac{u_{\varphi}^{2}}{r}+u_{z} \frac{\partial u_{r}}{\partial z}=-\frac{1}{\rho} \frac{\partial p}{\partial r}+\nu \frac{\partial}{\partial r}\left[\frac{1}{r} \frac{\partial}{\partial r}\left(r u_{r}\right)\right]+\nu \frac{\partial^{2} u_{r}}{\partial z^{2}}
\end{gathered}
$$




$$
\begin{aligned}
& u_{r} \frac{\partial u_{\varphi}}{\partial r}+\frac{u_{r} u_{\varphi}}{r}+u_{z} \frac{\partial u_{r}}{\partial z}=\nu \frac{\partial}{\partial r}\left[\frac{1}{r} \frac{\partial}{\partial r}\left(r u_{\varphi}\right)\right]+\nu \frac{\partial^{2} u_{\varphi}}{\partial z^{2}} \\
& u_{r} \frac{\partial u_{z}}{\partial r}+u_{z} \frac{\partial u_{z}}{\partial z}=-\frac{\partial p}{\partial z}+\nu\left[\frac{1}{r} \frac{\partial}{\partial r}\left(r \frac{\partial u_{z}}{\partial r}\right)\right]+\nu \frac{\partial^{2} u_{z}}{\partial z^{2}} .
\end{aligned}
$$

These simplified equations can still not be solved analytically, which suggests an order-of-magnitude analysis to allow further simplifications. For this purpose, the characteristic length scales in azimuthal and radial direction are the gap height $h$ and the mean radius $R=\frac{R_{1}+R_{2}}{2}$, respectively. Similarly, the characteristic velocities in azimuthal and radial direction are $\Omega R$ and $q / R$, where $q=Q / 2 \pi h$ is the volume flow rate per unit gap height. The quantities $q$ and $\Omega$ are intensive, as they do not depend on geometrical properties of the system, and are constant throughout the radius for purely radial and rotatory flows, respectively.

Inclusion of these characteristic quantities into the simplified continuity equation (8) yields

$$
\mathcal{O}\left(u_{z}\right) \propto \mathcal{O}\left(\frac{q}{R} \frac{h}{R}\right)
$$

which is an estimate of the order of magnitude of $u_{z}$.

The order of magnitude analyses for the momentum conservation in radial, circumferential and axial direction yield

$$
\begin{aligned}
& \overbrace{u_{r} \frac{\partial u_{r}}{\partial r}}^{\mathcal{O}\left(\frac{q^{2}}{R^{3}}\right)}-\overbrace{\frac{u_{\varphi}^{2}}{r}}^{\mathcal{O}\left(\Omega^{2} R\right)}+\overbrace{u_{z} \frac{\partial u_{r}}{\partial z}}^{\mathcal{O}\left(\frac{q^{2}}{R^{3}}\right)}=-\frac{1}{\rho} \frac{\partial p}{\partial r}+\overbrace{\nu \frac{\partial}{\partial r}\left[\frac{1}{r} \frac{\partial}{\partial r}\left(r u_{r}\right)\right]}^{\mathcal{O}\left(\nu \frac{q}{R^{3}}\right)}+\overbrace{\nu \frac{\partial^{2} u_{r}}{\partial z^{2}}}^{\mathcal{O}\left(\nu \frac{q}{h^{2} R}\right)}, \\
& \overbrace{u_{r} \frac{\partial u_{\varphi}}{\partial r}}^{\mathcal{O}\left(\frac{q \Omega}{R}\right)}+\overbrace{\frac{u_{r} u_{\varphi}}{r} u_{z} \frac{\partial u_{\varphi}}{\partial z}}^{\mathcal{O}\left(\frac{q \Omega}{R}\right)}=\overbrace{\nu \frac{\partial}{\partial r}\left[\frac{1}{r} \frac{\partial}{\partial r}\left(r u_{\varphi}\right)\right]}^{\mathcal{O}\left(\frac{q \Omega}{R}\right)}+\overbrace{\nu \frac{\partial^{2} u_{\varphi}}{\partial z^{2}}}^{\mathcal{O}\left(\nu \frac{\Omega}{R}\right)}, \\
& \overbrace{u_{r} \frac{\partial u_{z}}{\partial r}}^{\mathcal{O}\left(\frac{q^{2} h}{R^{4}}\right)}+\overbrace{u_{z} \frac{\partial u_{z}}{\partial z}}^{\mathcal{O}\left(\frac{q^{2} h}{R^{4}}\right)}=-\frac{\partial p}{\partial z}+\overbrace{\nu\left[\frac{1}{r} \frac{\partial}{\partial r}\left(r \frac{\partial u_{z}}{\partial r}\right)\right]}^{\mathcal{O}\left(\nu \frac{q h}{R^{4}}\right)}+\overbrace{\nu \frac{\partial^{2} u_{z}}{\partial z^{2}}}^{\mathcal{O}\left(\nu \frac{q}{R^{2} h}\right)},
\end{aligned}
$$

where the estimate of $\mathcal{O}\left(u_{z}\right)$ as obtained from the continuity equation (12) has already been exploited. 
In order to better assess the relative importance of the respective terms, the order of magnitude analysis is rewritten so that the first term on the left-hand side of Eqs. (13)- 15$)$ is of order $\mathcal{O}\left(q h^{2} / \nu R^{2}\right)$. This is achieved by multiplication of Eq. (13) with $\mathcal{O}\left(R h^{2} / q \nu\right)$, Eq. (14) with $\mathcal{O}\left(h^{2} / \Omega R \nu\right)$ and Eq. 15 with $\mathcal{O}\left(R^{2} h / \nu\right)$, to obtain:

$$
\begin{aligned}
& \overbrace{u_{r} \frac{\partial u_{r}}{\partial r}}^{\mathcal{O}\left(\frac{q}{\nu} \frac{h^{2}}{R^{2}}\right)}-\overbrace{\frac{u_{\varphi}^{2}}{r}}^{\mathcal{O}\left(\frac{\Omega^{2} R^{2} h^{2}}{q \nu}\right)}+\overbrace{u_{z} \frac{\partial u_{r}}{\partial z}}^{\mathcal{O}\left(\frac{q}{\nu} \frac{h^{2}}{R^{2}}\right)}=-\frac{1}{\rho} \frac{\partial p}{\partial r}+\overbrace{\nu \frac{\partial}{\partial r}\left[\frac{1}{r} \frac{\partial}{\partial r}\left(r u_{r}\right)\right]}^{\mathcal{O}\left(\frac{h^{2}}{R^{2}}\right)}+\overbrace{\nu \frac{\partial^{2} u_{r}}{\partial z^{2}}}^{\mathcal{O}(1)}, \\
& \overbrace{u_{r} \frac{\partial u_{\varphi}}{\partial r}}^{\mathcal{O}\left(\frac{q}{\nu} \frac{h^{2}}{R^{2}}\right)}+\overbrace{\frac{u_{r} u_{\varphi}}{r}}^{\mathcal{O}\left(\frac{q}{\nu} \frac{h^{2}}{R^{2}}\right)} \overbrace{u_{z} \frac{\partial u_{\varphi}}{\partial z}}^{\mathcal{O}\left(\frac{q}{\nu} \frac{h^{2}}{R^{2}}\right)}=\overbrace{\nu \frac{\partial}{\partial r}\left[\frac{1}{r} \frac{\partial}{\partial r}\left(r u_{\varphi}\right)\right]}^{\mathcal{O}\left(\frac{h^{2}}{R^{2}}\right)}+\overbrace{\nu \frac{\partial^{2} u_{\varphi}}{\partial z^{2}}}^{\mathcal{O}(1)} \\
& \overbrace{u_{r} \frac{\partial u_{z}}{\partial r}}^{\mathcal{O}\left(\frac{q}{\nu} \frac{h^{2}}{R^{2}}\right)}+\overbrace{u_{z} \frac{\partial u_{z}}{\partial z}}^{\mathcal{O}\left(\frac{q}{\nu} \frac{h^{2}}{R^{2}}\right)}=-\frac{\partial p}{\partial z}+\overbrace{\nu\left[\frac{1}{r} \frac{\partial}{\partial r}\left(r \frac{\partial u_{z}}{\partial r}\right)\right]}^{\mathcal{O}\left(\frac{h^{2}}{R^{2}}\right)}+\overbrace{\nu \frac{\partial^{2} u_{z}}{\partial z^{2}}}^{\mathcal{O}(1)} .
\end{aligned}
$$

Assuming that $h / R \ll 1$, the first term on the right-hand side of Eqs. (13)-15) can be considered negligible. Likewise, all nonlinear terms on the left-hand side of Eqs. (13)-(15), except the second term of Eq. (13), can be neglected according to the assumption that the radial convection is small compared to viscous diffusion, i.e.

$$
R e_{q}=\frac{q h}{\nu R} \ll \frac{R}{h} .
$$

$R e_{q}$ is the Reynolds number for the radial flow, which can also be extracted from the dimensionless numbers $(3)$. Introduction of the ratio

$$
\alpha=\frac{q}{\Omega R^{2}}
$$

between the characteristic radial and azimuthal characteristic velocities into Eq. (19) recasts $R e_{q}$ in terms of a lubrication Reynolds number $R e_{l}$ as

$$
R e_{l} \ll \frac{R}{h} \frac{1}{\alpha}
$$


The assumption of Eq. (21) and its equivalent Eq. (19) are similar to the one typically adopted in the derivation of the Reynolds equation for the quasi-parallel flow between two slightly inclined walls. Differently from the Reynolds equation, the deviation from the parallel condition is due to the relative importance of the imposed radial flow, which is measured by $\alpha$, rather than being of purely geometric nature. For the present problem, $\alpha \ll 1$ holds.

The second term in Eq. (13) represents the centrifugal forces. Its relative importance compared to the other terms of the equation can be estimated by elimination of either $q$ or $\Omega$ through the definition of Eq. (20), which yields

$$
\mathcal{O}\left(\frac{\Omega^{2} R^{2} h^{2}}{q \nu}\right)=\mathcal{O}\left(R e_{q} \frac{h}{R \alpha^{2}}\right)=\mathcal{O}\left(R e_{l} \frac{h}{R \alpha}\right)
$$

Due to $\alpha$ appearing at the denominator, the second term on the left-hand side of Eq. (13) can not be neglected whenever the rotational motion is predominant.

In consequencs, the dimensional analysis leads to the following simplified system of differential equations

$$
\begin{gathered}
\frac{1}{r} \frac{\partial\left(r u_{r}\right)}{\partial r}+\frac{\partial u_{z}}{\partial z}=0 \\
-\frac{u_{\varphi}^{2}}{r}=-\frac{1}{\rho} \frac{\partial p}{\partial r}+\nu \frac{\partial^{2} u_{r}}{\partial z^{2}} \\
0=\nu \frac{\partial^{2} u_{\varphi}}{\partial z^{2}} \\
0=-\frac{\partial p}{\partial z}+\nu \frac{\partial^{2} u_{z}}{\partial z^{2}}
\end{gathered}
$$

and their respective boundary conditions for the present flow

$$
\begin{array}{r}
u_{r}(r, \varphi, z=h)=0 ; u_{r}(r, \varphi, z=0)=0 \\
u_{\varphi}(r, \varphi, z=h)=r \Omega ; u_{\varphi}(r, \varphi, z=0)=0 \\
u_{z}(r, \varphi, z=h)=0 ; u_{z}(r, \varphi, z=0)=0 .
\end{array}
$$

It is possible to solve the system analytically, once it is shown that $\partial p / \partial r$ is not a function of $z$. This is easily achieved by performing the same orderof-magnitude analysis for the difference between the $z$-derivative of Eq. (9) 
and the $r$-derivative of Eq. (11), which results in

$$
-\frac{\partial u_{\varphi}^{2}}{\partial z}=\nu \frac{\partial^{3} u_{r}}{\partial z^{3}}
$$

Integration of Eq. (30) in the $z$-direction yields

$$
-\frac{u_{\varphi}^{2}}{z}=+f(r)+\nu \frac{\partial^{2} u_{r}}{\partial z^{2}}
$$

Consideration of a representative set of realistic parameters (as listed in Table 1) illustrates the ratio of the volume flow rate $Q$ regarding these

It is important to note that the solution for the radial velocity of Eq. (32) requires, together with the continuity equation, that $u_{z} \neq 0$.

The pressure gradient $\Pi$ is unknown in Eq. (32) and has to be determined by an additional equation. In the present case the volumetric flow rate $Q$ is a known parameter and an integration of the radial velocity with regard to $z$ and $\varphi$ yields

$$
Q=\int_{0}^{2 \pi} \int_{0}^{h} u_{r} r \mathrm{~d} z \mathrm{~d} \varphi=-\frac{\pi h^{3}}{6 \mu} \Pi+\frac{\pi r^{2} h^{3} \Omega^{2}}{20 \nu}
$$

which leaves the pressure gradient in radial direction as the only remaining unknown. The first term on the right-hand side is associated with the pressure driven Poiseuille flow. The second term represents the flow as induced by centrifugal forces, which act on the fluid due to the rotational speed $\Omega$. Eq. (34) can be rewritten as follows:

$$
\Pi=\frac{\partial p}{\partial r}=-\frac{6 \mu Q}{\pi h^{3} r}+\frac{3 \rho \Omega^{2} r}{10} .
$$


Table 1: Geometric dimensions used for further calculations

\begin{tabular}{l|c|l}
\hline Parameter & Symbol & Values \\
\hline inner radius of the disk & $R_{1}$ & $8.25 \cdot 10^{-2}[\mathrm{~m}]$ \\
outer radius of the disk & $R_{2}$ & $9.375 \cdot 10^{-2}[\mathrm{~m}]$ \\
feeding oil volume flow rate & $Q$ & $1.667 \cdot 10^{-5}\left[\mathrm{~m}^{3} / \mathrm{s}\right]$ \\
density & $\rho$ & $850\left[\mathrm{~kg} / \mathrm{m}^{3}\right]$ \\
dynamic viscosity & $\mu$ & $0.0136[\mathrm{~Pa} \mathrm{~s}]$ \\
kinematic viscosity & $\nu$ & $16 \cdot 10^{-6}\left[\mathrm{~m}^{2} / \mathrm{s}\right]$ \\
temperature & $T$ & $40\left[{ }^{\circ} \mathrm{C}\right]=\mathrm{const}$ \\
gap height & $h$ & $250 \cdot 10^{-6}[\mathrm{~m}]$ \\
angular velocity & $\Omega$ & $0<\Omega<314[1 / \mathrm{s}]$ \\
& & $\widehat{=} 0<n<3000[\mathrm{rpm}]$ \\
\hline
\end{tabular}

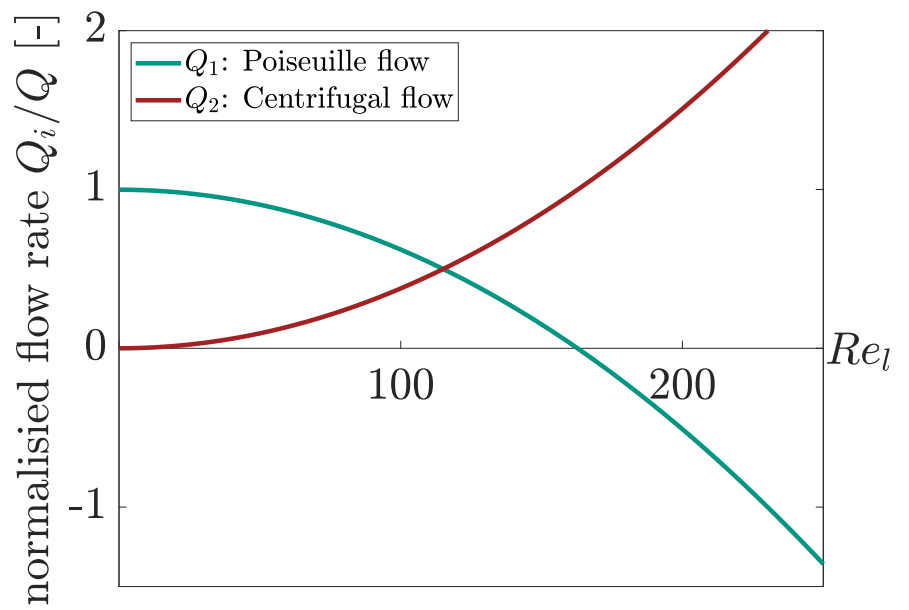

Figure 2: Ratio of normalized volume flow rate $Q_{i} / Q$ as a function of lubrication Reynolds number $R e_{l}$, where $i=1,2$ corresponds to Poiseuille and centrifugal flow contributions; note that $Q_{1}+Q_{2}=Q$ for all parameter combinations.

two terms of Eq. (34) as a function of lubrication Reynolds number $R e_{l}=$ $\Omega R_{2} h / \nu$ at radial position $r=R_{2}$; see Figure 2 .

It is obvious that an increasing lubrication Reynolds number leads to an 165 increased contribution of the centrifugal flow to the total volume flow rate. In consequence the Poiseuille-flow contribution and thus the radial pressure gradient is reduced. With a known pressure gradient as a function of $\Omega$ it is possible to calculate the corresponding circumferential and radial velocity 
profiles. Figure 3 shows the radial velocity profile based on the parameters 170 of Table 1. The increasing relevance of the centrifugal forces in the upper half of the gap with increasing $R e_{l}$ can be clearly identified from the salient shift of the velocity maximum from the gap center towards the rotor. If the angular velocity is increased further, the centrifugal force becomes the most dominant part in the equation. At $R e_{l}=113$ the pressure gradient changes sign and at $R e_{l}=245$ the wall shear stress in radial direction changes sign at $z=0$.

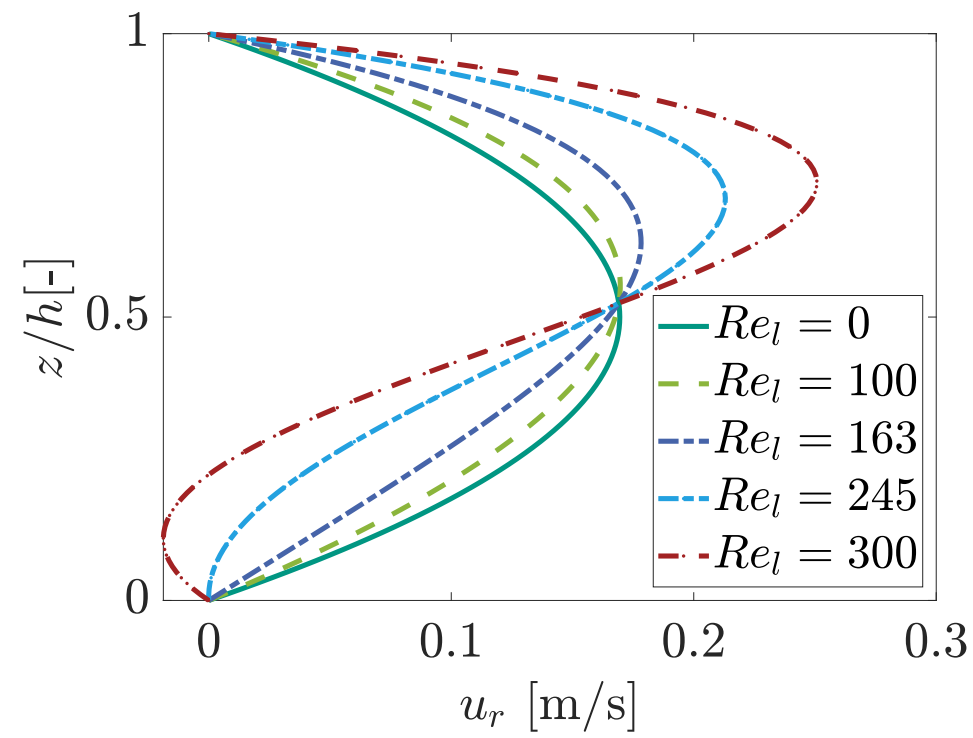

Figure 3: Velocity component $u_{r}$ for five different Reynolds numbers at $r=R_{2}$ for the parameters shown in Table 1

\subsection{Drag torque and aeration equations}

One common class of aeration models [8, 10] uses the sign change of the pressure gradient at $r=R_{2}$ as onset for aeration

$$
0 \stackrel{!}{=} \Pi\left(r=R_{2}, \varphi, z\right)=-\frac{6 \mu Q}{\pi h^{3} R_{2}}+\frac{3 \Omega_{c r i t}^{2} R_{2}}{10} .
$$

In addition to the aeration onset these models aim at predicting the drag torque. The most straightforward way for its calculation is a spatial integration of the shear stress (1) at one of the disks, which is directly given by the 
velocity profiles derived in Eq. (33) according to

$$
T_{s}=\int_{A} \tau_{\varphi z} r \mathrm{~d} A=2 \pi \int_{R_{1}}^{R_{2}} \mu\left(\frac{\Omega r}{h}\right) r^{2} \mathrm{~d} r=\frac{\pi \mu \Omega}{2 h}\left(R_{2}^{4}-R_{1}^{4}\right) .
$$

The simplification of the governing equations, as discussed above, provides an analytical solution for the velocity profiles in radial and circumferential direction. With these two equations a calculation of the drag torque can be done, which also allows an estimation of the operating point, where the air enters the system. These equations are used to define a dimensionless relationship in the following section.

\section{Results of the dimensional analysis}

The dimensionless numbers (3) as derived in Section 2, can be used to define a more general criterion of the torque model and the aeration onset. Using the aeration model, where the onset occurs at $\partial p / \partial r=0$ as discussed in Section 2.2 leads to

$$
\begin{aligned}
& 0 \stackrel{!}{=} \Pi(r\left.=R_{2}, \varphi, z\right)=-\frac{6 \mu Q}{\pi h^{3} R_{2}}+\frac{3 \Omega_{c r i t}^{2} R_{2}}{10} \\
& \Leftrightarrow Q_{c r i t}^{*}=\frac{Q \nu}{\Omega_{c r i t}^{2} R_{2}{ }^{2} h^{3}}=\frac{\pi}{20} .
\end{aligned}
$$

This indicates that aeration does not occur while

$$
\frac{Q \nu}{\Omega^{2} R_{2}^{2} h^{3}}=Q^{*}>Q_{c r i t}^{*}=\frac{\pi}{20} .
$$

Consequently, aeration is expected to occur for operating conditions with dimensionless flow rate below this critical value.

The analytical solution of the velocity field can also be used to obtain a relation between $\zeta_{m}$ and $R e_{l}$ according to

$$
\begin{gathered}
\zeta_{m}=\frac{T_{s}}{\frac{\rho}{2} R_{2}{ }^{5} \Omega^{2}\left(1-\beta^{4}\right)}=\frac{\frac{\pi \mu \Omega}{2 h}\left(R_{2}{ }^{4}-R_{1}{ }^{4}\right)}{\frac{\rho}{2} R_{2}{ }^{5} \Omega^{2}\left(1-\beta^{4}\right)} \\
\Leftrightarrow \zeta_{m}=\frac{\pi}{R e_{l}} .
\end{gathered}
$$


This relation describes the dependence of the drag torque on the operating conditions before the onset of aeration. Thus the dimensionless reformulation of the simplified governing equations provides two simple correlations that allow to directly estimate drag torque and aeration onset for any open wet clutch flow, given the applied simplifications are justified.

A simple example is chosen to demonstrate the benefit of the dimensionless approach in the following. Figure 4 depicts the drag torque for three geometric parameter combinations in terms of gap height $h$, volumetric flow rate $Q$ and outer radius $R_{2}$. For all three parameter combinations it is possible to calculate the corresponding slope of the drag torque and the point, where aeration occurs, according to Eq. (37) and Eq. (36), respectively. Consequently, this leads to three different slopes and three different aeration points. Converted to the above-derived dimensionless quantities the three different parameter combinations collapse onto a single curve in a $\zeta_{m}-R e_{l}$ diagram according to Eq. (38); see Figure $5 \mathrm{a}$.

The markers in Figure 4 represent the points at which aeration occurs according to Eq. (36). For the chosen different parameter combinations this happens at three different values of $\Omega$. Similarly normalized, all points collapse for a non-dimensional critical flow rate $Q_{c r i t}^{*}=\frac{\pi}{20} \approx 0.157$, as shown in Figure $5 \mathrm{~b}$. On the ordinate of this diagram the combined quantity $\zeta_{m} R e_{l} / \pi$ is plotted. Since the analytical relations in Fig. 5 are valid for the idealized flow situation where all applied simplifications are fully justified, it is expected that experimental or numerical results might show some deviations. Note, however, that the order of magnitude for maximum drag torque before aeration onset can likewise be captured with any existing model [7, 8, 9, 11, 20]. So the dimensionless approach isn't limited to a specific model.

Another benefit is the possibility to compare different experimental drag torque measurements obtained on test rigs with different geometric dimensions. The markers in Figure 6 indicate the point of aeration from different publications.

All considered experimental data [12, 14] match the theoretical prediction according to Eq. (38) in the $\zeta_{m}-R e_{l}$ diagram (Figure 6a), which retroactively also verifies Eq. (37) as to be an appropriate drag-torque prediction.

Figure $6 \mathrm{~b}$ shows the product of $\zeta_{m} R e_{l} / \pi$ over the normalized flow rate $Q^{*}$. The theoretical value for aeration is placed at $\pi / 20$, if the above-outlined assumption of a sign change of the pressure gradient as to coincide with the aeration onset is considered. The values are in the same order-of-magnitude, but deviate considerably from the theoretical value. Two possible reasons 


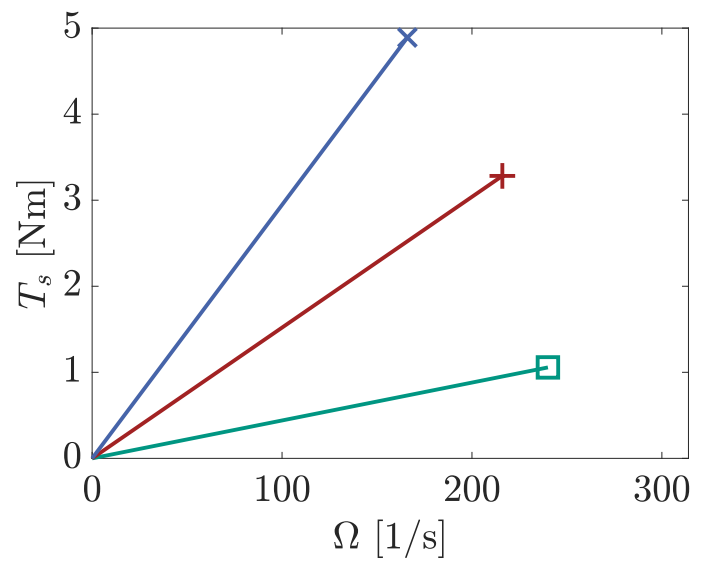

Figure 4: Drag torque $T_{s}$ over angular velocity for three different parameter combinations: 口: $h=150 \mu \mathrm{m}, Q=1 \mathrm{l} / \mathrm{min}, R_{2}=0.09375 \mathrm{~m}$;

口 : $h=200 \mu \mathrm{m}, Q=3 \mathrm{l} / \mathrm{min}, R_{2}=0.1172 \mathrm{~m}$;

口 $h=250 \mu \mathrm{m}, Q=5 \mathrm{l} / \mathrm{min}, R_{2}=0.1402 \mathrm{~m}$

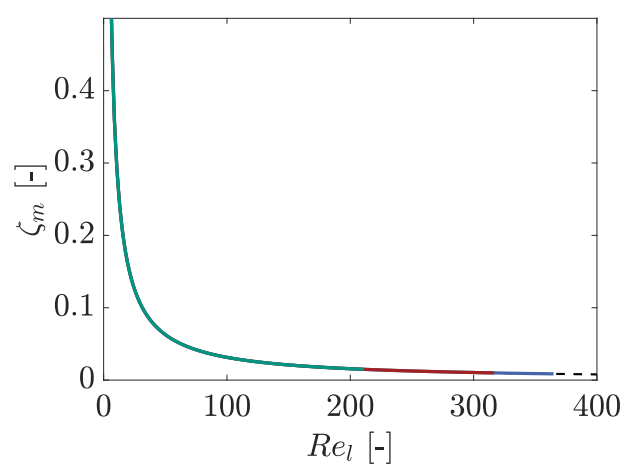

(a)

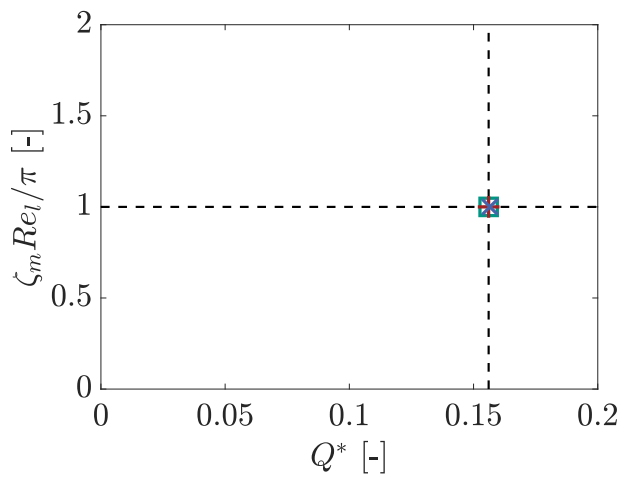

(b)

Figure 5: (a) Moment coefficient $\zeta_{m}$ over lubrication Reynolds number $R e_{l}$ and (b) Product $\zeta_{m} R e_{l} / \pi$ over $Q^{*}$ of the parameter combinations used in Figure 4 .

might cause this deviation. Either the theoretical description for the aeration onset is insufficient or the assumptions used for the derivations oversimplify this type of experimental flow. A broader experimental data set would be required to testify and clarify the latter, which is beyond the scope of the present work. As for the former, however, the fact that the drag torque fits well to the assumptions made, indicates an imprecise condition for the aeration onset. From a fluid dynamic point of view a reverse flow, which isn't 
identical with a sign change of the pressure gradient for this flow, is more likely to lead to aeration. For this flow configuration a reverse flow at $z=0$ occurs at $Q^{*}=\frac{\pi}{45}$, according to the partial derivation of Eq. (32).

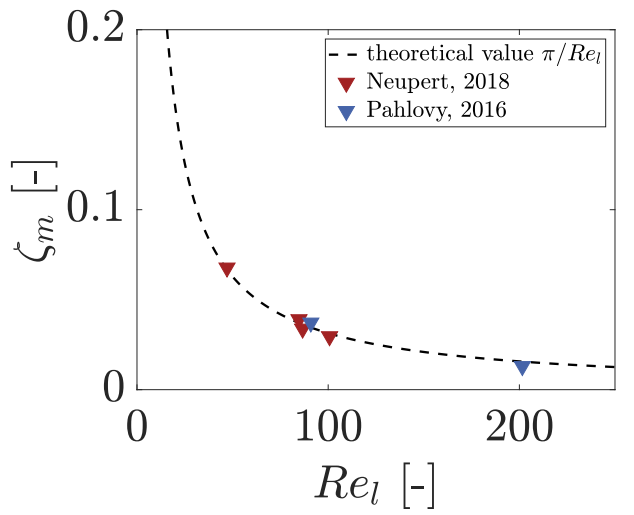

(a)

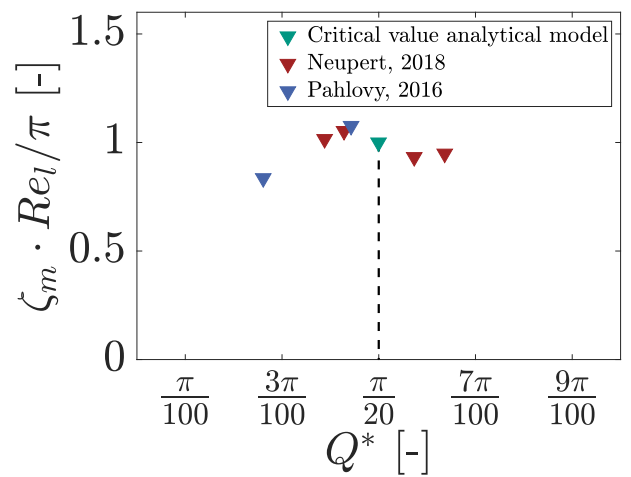

(b)

Figure 6: Comparison of the drag torque coefficient (a) and aeration onset (b) for an ungrooved disk obtained from experimental data from [12, 14].

\section{Effect of grooves on aeration onset}

The fact that grooves can yield an aeration onset at lower circumferential speed as compared to a flat disk, has been discussed by various authors as has been recently summarized by NEUPERT et al. [14, 21], for instance.

Figure 7 shows a radially grooved disk, which comprises the relevant geometrical parameters. PAHLOVy et al. [11] introduce the equivalent gap height value for a grooved disk as

$$
h_{\text {eq,pah }}=h+H a_{g}=h+H \frac{A_{\text {groove }}}{A_{\text {disk }}} .
$$

Here, $a_{g}$ is the ratio of the grooved and ungrooved surface area and $H$ is the height of the groove. The equivalent gap height $h_{e q, p a h}$ is thus a weighted mean of the grooved and ungrooved areas. This leads consequently to an earlier aeration onset. Even tough the modelling approach shows promising results in comparison with experiments for a specific groove geometry, it is not capable to capture the complex non-linear behaviour of different types of grooves as examined by [14]. Especially the expected effect of the change 


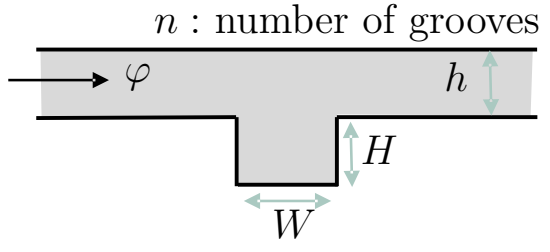

(a) Groove segment of a radial groove area $A$; - wetted perimeter $P$

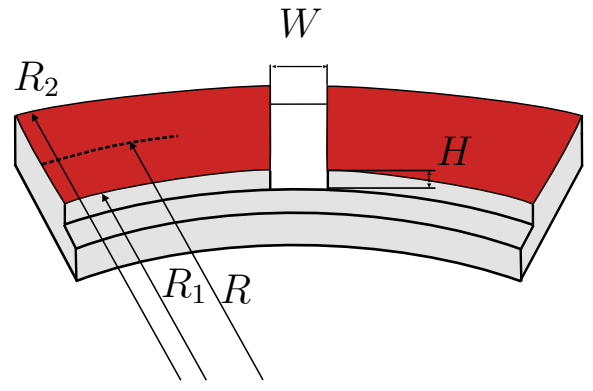

(b) Perspective scheme of a radial groove segment; $\square$ grooved area $A_{\text {groove, }} \square$ ungrooved area $A_{d i s k}$.

Figure 7: Geometric dimensions and designation of the disk and groove.

of groove ratio $H / W$ (where $W$ is the groove width) cannot be considered properly with this approach as shown later.

Inspired by these earlier efforts, an alternative formulation of the equivalent gap height is introduced in the following, which builds upon the hydraulicdiameter concept.

The forces that act on an infinite fluid volume moving from the inner to the outer radius of an open clutch can be divided into separate constitutive components. For a smooth disk this summation of forces can be modelled based on the simplified Navier-Stokes equations discussed earlier. The point of aeration is mainly a balance between inertia, centrifugal and frictional forces. The introduction of grooves moves this balance to another equilibrium state. Increasing the cross-sectional through-flow area at the same pressure gradient reduces the frictional forces on one hand. Frictional forces are, on the other hand, increased with a greater wetted perimeter.

The analytical solution based on the assumptions for smooth disks are not suitable for grooved surfaces, since they cannot consider the complex flow phenomena in the grooves, such as e.g. helical and/or vortical structures inside the grooves [22]. A profound analysis of the underlying fluid flow must be done by experiments or numerical simulations. Nevertheless, for a first and fast approximation an adapted version of the model for smooth walls might be able to deliver acceptable results. An easy way to capture a complex geometry in fluid mechanics is the concept of hydraulic diameter, originally introduced for turbulent flow in ducts with a non-circular cross-section (see e.g. [23]). The hydraulic diameter for the present flow can be calculated with the cross sectional area $A$ at the mean radius $R$ and the wetted perimeter of 
the cross-section $P$

$$
D_{h y}=\frac{4 A}{P}
$$

where

$$
A=2 \pi R h \text { and } P=4 \pi R ; \quad R=\frac{R_{1}+R_{2}}{2} .
$$

For an ungrooved disk this value gives $D_{h y}=2 h$. For a radially grooved disk $A$ and $P$ both change such that the equivalent gap height can be defined as

$$
h_{e q}=\frac{D_{h y}}{2}=\frac{2 A}{P}=\frac{h \pi\left(R_{1}+R_{2}\right)+n H W}{\pi\left(R_{1}+R_{2}\right)+n H} .
$$

Reorganisation of Eq. (41) leads to

$$
h_{e q}=\frac{h+H \frac{A_{\text {groove }}}{A_{\text {disk }}}}{1+\frac{H}{W} \frac{A_{\text {groove }}}{A_{\text {disk }}}} .
$$

The direct comparison with Eq. (39) as suggested by PAHLOVY et al. [11] indicates that the numerators of both approaches are identical. However, differences exist in the denominator, where the present model formulation introduces a non-linear influence of the groove height. Figure 8 shows the direct comparison of these two approaches, Eq. $(39)$ and Eq. 42 , to indicate the influence of the modified denominator i.e. the impact of the hydraulic diameter. Figure $8 \mathrm{a}$ and $8 \mathrm{~b}$ depict $h_{e q}$ as a function of groove height $H$ for constant groove width $W$ and vice versa, respectively. Figure $8 \mathrm{c}$ and 8d represent slightly more complex variation scenarios. The third diagram shows a variation of $W$ and $H$, while the product of these two quantities is constant. For the fourth diagram the frictional area is set constant, so the number of grooves $n$ decreases with increasing width $W$. The latter two cases are chosen, to provide comparability to the systematic experimental study of Neupert et al. [14. The advantages of the hydraulic diameter approach becomes obvious from the latter two cases (Figs. 8c and 8d). For a constant product of groove height and width the model based on Eq. (39) does not predict a change in drag torque, whereas the new approach, Eq. (42), offers a systematic dependency, which is in agreement with the experimental findings. This different character also holds for a constant frictional area, which is the fourth case. 


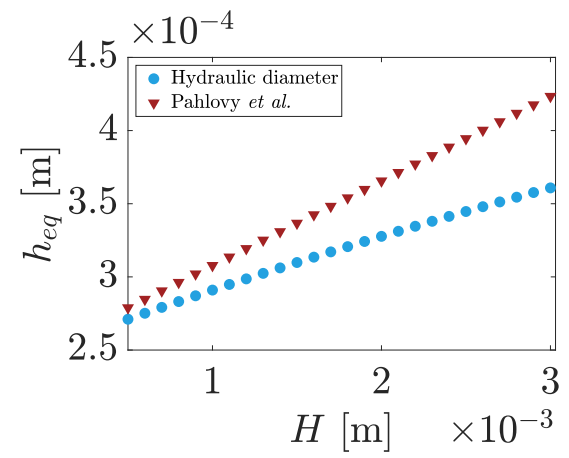

(a) $W=1 \mathrm{~mm} ; n=32$

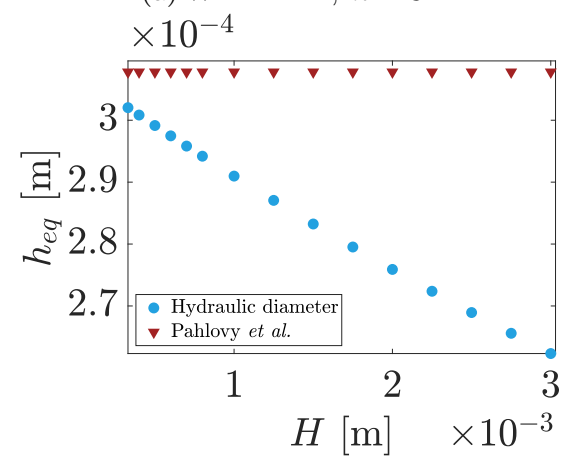

(c) $W \cdot H=1 \mathrm{~mm}^{2}=$ const; $n=32$

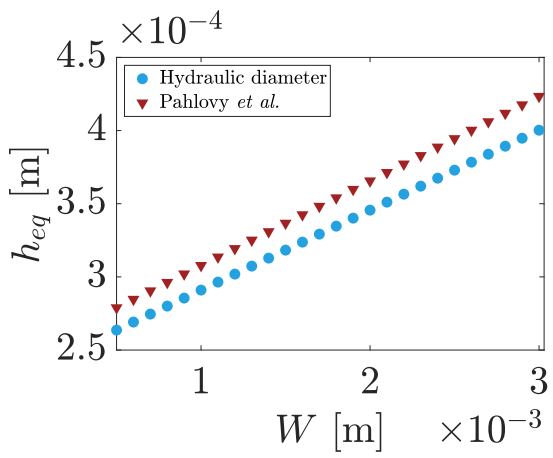

(b) $H=1 \mathrm{~mm} ; n=32$

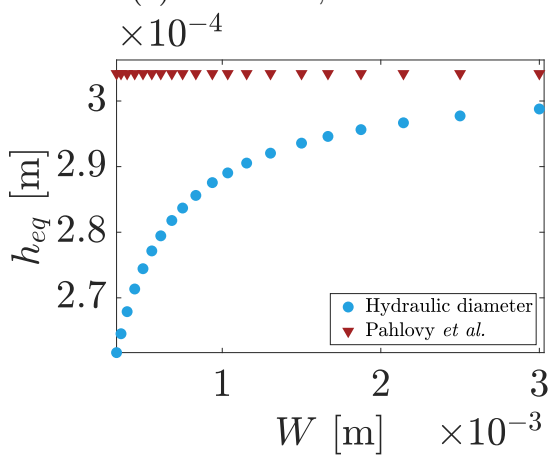

(d) $H=1 \mathrm{~mm} ; A_{\text {disk }}=$ const.

Figure 8: A systematic comparison of both evaluated models with different parameters. - : present approach using hydraulic diameter concept; $\mathbf{\nabla}$ : approach of PAHLOVY et al. [11]

\section{Conclusion}

The simplifications required to obtain an analytical solution of the mass and momentum conservation equations for the fluid flow in open wet clutch flows with smooth disks have been discussed in detail, so as to provide a basis to derive a precise analytical model for drag-torque and aeration-onset predictions. It is shown that the momentum conservation equations reduce to two independent ordinary differential equations in radial and circumferential direction for flow cases in which $\frac{u_{r} h^{2}}{\nu R} \ll 1$ is justified. It has been demonstrated that the simplifications made by existing open clutch flow models are appropriate as long as this assumption is valid.

A dimensional analysis of the obtained equations has been done to reduce the problem to its principle degrees of freedom. Particularly, a single equation has been identified to predict the normalized drag torque as a function 
of lubrication Reynolds number, which holds for a variety of possible operating conditions and clutch geometries. It has further been demonstrated that this equation agrees with the simplified version of the governing equations. This insight, in turn, provides evidence that the derived dimensionless quantities might serve as a more robust and universal metric to describe and consequently also predict the drag torque characteristics across the board of parameter combinations.

Furthermore, the dimensionless aeration onset can be formulated as a constant number, which takes the value of $\pi / 20$ for the existing aeration models. It is therefore recommended that experimental data for aeration onset obtained in different test rigs with different geometric dimensions and inherent test-rig particularities should be compared in the dimensionless $\zeta_{m} R e_{l} / \pi$ $Q^{*}$ - diagram, where deviations from the idealized and simplified model formulation are directly visible.

Additionally, a new modelling concept for the influence of radial grooves in open wet clutch flows based on the hydraulic diameter concept is introduced. This new approach advances beyond earlier concepts, since the formerly missing effect of the groove aspect ratio $(H / W)$ is considered. It can be, therefore, concluded that the new approach supplies a more accurate prediction due to a more detailed consideration of the ongoing phenomena in the lubrication gap.

Finally, in combination with the dimensionless approach the hydraulicdiameter concept might serve as a new and broader basis to more accurately predict the drag force and the rotational velocity for the onset of aeration in open wet clutches.

\section{References}

[1] Frederick Lloyd. Parameters contributing to power loss in disengaged wet clutches. In SAE Technical Paper Series. SAE International, 1974.

[2] Y. Kato, T. Murasugi, H. Hirano, and T. Shibayama. Fuel economy improvements through tribological analysis of the wet clutches and brakes of an automatic transmission. In Society of Automotive Engineers of Japan, volume 16, pages 57-60, 1993.

[3] H. Hashimoto, S. Wada, and Y. Murayama. The performance of a turbulent-lubricated sliding bearing subject to centrifugal effects. In 
Trans. Jpn, Society Mechanical Engineering, volume 49, pages 17531761, 1984.

[4] Yiqing Yuan, Eysion A. Liu, James Hill, and Qian Zou. An improved hydrodynamic model for open wet transmission clutches. Journal of Fluids Engineering, 129(3):333, 2007.

[5] Brian Launder, Sébastien Poncet, and Eric Serre. Laminar, transitional, and turbulent flows in rotor-stator cavities. Annual Review of Fluid Mechanics, 42(1):229-248, 2010.

[6] Hisanao Kitabayashi, Chen $\mathrm{Yu} \mathrm{Li}$, and Henry Hiraki. Analysis of the various factors affecting drag torque in multiple-plate wet clutches. In SAE Technical Paper Series. SAE International, 2003.

[7] Guang Rao. Modellierung und Simulation des Systemverhaltens nasslaufender Lamellenkupplungen. Dissertation, Technische Universität Dresden, Dresden, 2010.

[8] Jiahai Huang, Jianhua Wei, and Minxiu Qiu. Laminar flow in the gap between two rotating parallel frictional plates in hydro-viscous drive. Chinese Journal of Mechanical Engineering, 25(1):144-152, 2012.

[9] Shoaib Iqbal, Farid Al-Bender, Bert Pluymers, and Wim Desmet. Mathematical model and experimental evaluation of drag torque in disengaged wet clutches. ISRN Tribology, 2013:16, 2013.

[10] Shoaib Iqbal, Farid Al-Bender, Bert Pluymers, and Wim Desmet. Model for predicting drag torque in open multi-disks wet clutches. Journal of Fluids Engineering, 136(2):021103, 2013.

[11] Shahjada Ahmed Pahlovy, Syeda Faria Mahmud, Masamitsu Kubota, Makoto Ogawa, and Norio Takakura. Multiphase drag modeling for prediction of the drag torque characteristics in disengaged wet clutches. SAE International Journal of Commercial Vehicles, 7(2):441-447, 2014.

[12] Shahjada Ahmed Pahlovy, Syeda Faria Mahmud, Masamitsu Kubota, Makoto Ogawa, and Norio Takakura. Prediction of drag torque in a disengaged wet clutch of automatic transmission by analytical modeling. Tribology Online Japanes Society of Tribologists, 11(2):121-129, 2016. 
[13] Shahjada Ahmed Pahlovy, Syeda Faria Mahmud, Masamitsu Kubota, Makoto Ogawa, and Norio Takakura. Development of an analytical model for prediction of drag torque characteristics of disengaged wet clutches in high speed region. In SAE Technical Paper Series, SAE Technical Paper. SAE International400 Commonwealth Drive, Warrendale, PA, United States, 2017.

[14] Thomas Neupert, Elisabeth Benke, and Dirk Bartel. Parameter study on the influence of a radial groove design on the drag torque of wet clutch discs in comparison with analytical models. Tribology International, 119:809-821, 2018.

[15] Leonid P. Yarin. The Pi-theorem : applications to fluid mechanics and heat and mass transfer. Experimental fluid mechanics. Springer, Berlin, 2012 .

[16] Jürgen Zierep. Ähnlichkeitsgesetze und Modellregeln der Strömungslehre. Braun-Verlag, 1992.

[17] J. M. Owen and R. H. Rogers. Flow and Heat Transfer in Rotating Disc Systems, Vol.1: Rotor-Stator Systems. Research Studies Press, 1989.

[18] Andrea Codrignani, Daniele Savio, Franco Magagnato, and Bettina Frohnapfel. A scaling parameter for pressure losses and thermal effects in lubricant flows with viscous dissipation. Tribology International, 113:238 - 244, 2017. 43rd Leeds - Lyon Symposium on Tribology.

[19] Daniel Gropper, Ling Wang, and Terry J. Harvey. Hydrodynamic lubrication of textured surfaces: A review of modeling techniques and key findings. Tribology International, 94:509-529, 2016.

[20] Hongwei Cui, Shouwen Yao, Qingdong Yan, Shanshan Feng, and Qian Liu. Mathematical model and experiment validation of fluid torque by shear stress under influence of fluid temperature in hydro-viscous clutch. Chinese Journal of Mechanical Engineering, 27(1):32-40, 2014.

[21] Thomas Neupert and Dirk Bartel. High-resolution 3d cfd multiphase simulation of the flow and the drag torque of wet clutch discs considering free surfaces. Tribology International, 129:283 - 296, 2019. 
400

[22] Robin Leister and Jochen Kriegseis. 3D-LIF experiments in an open wet clutch by means of defocusing PTV. In Christian J. Kähler, Rainer Hain, Sven Scharnowski, and Thomas Fuchs, editors, Proceedings of the 13th International Symposium on Particle Image Velocimetry, 2019.

[23] Hermann Schlichting and Klaus Gersten. Boundary-Layer Theory. Springer Berlin Heidelberg, 2017. 


\section{Appendix Analytical Models}

Over the last years a number of analytical solutions have been published. Four models are summarized in Table 2.

Table 2: Analytical models as discussed in the present work

\begin{tabular}{llll}
\hline No & Author & Year & Reference \\
\hline I & Rao & 2010 & {$[7]$} \\
II & Iqbal et al. & 2013 & {$[9],[10]$} \\
III & Pahlovy et al. & 2014,2016 & {$[11],[12]$} \\
IV & Huang et al. & 2012 & {$[8]$} \\
\hline
\end{tabular}

The simplifications made by every model are listed in the following simplified versions of the continuity and Navier-Stokes equations. The numbers under the braces correspond to the author numeration of Table 2 .

$$
\begin{gathered}
\underbrace{0=\frac{1}{r} \frac{\partial\left(r u_{r}\right)}{\partial r}}_{\text {I, II, III, IV }} \\
\rho(\underbrace{u_{r} \frac{\partial u_{r}}{\partial r}}_{\text {I, III }}-\underbrace{\frac{u_{\varphi}^{2}}{r}}_{\text {I, II, III, IV }})=-\underbrace{-\frac{\partial p}{\partial r}}_{\text {I, II, III, IV }}+\underbrace{\mu \frac{\partial^{2} u_{r}}{\partial z^{2}}}_{\text {I, II, III, IV }} \\
\rho(\underbrace{u_{r} \frac{\partial u_{\varphi}}{\partial r}}_{\text {I, III, IV }}+\underbrace{\frac{u_{r} u_{\varphi}}{r}}_{\text {I, III, IV }})=\underbrace{\mu \frac{\partial^{2} u_{\varphi}}{\partial z^{2}}}_{\text {I, II, III, IV }} \\
0=\underbrace{\frac{\partial p}{\partial z}}_{\text {I, II, III, IV }}
\end{gathered}
$$

This leads to the solutions of the radial and circumferential velocity, which can be written as 


$$
\begin{aligned}
u_{r}= & \underbrace{\frac{1}{2 \mu} \frac{\mathrm{d} p}{\mathrm{~d} r} z(z-h)}_{\text {I, II, III, IV }}+\underbrace{\frac{r \Omega^{2}}{12 \nu h^{2}}\left(h^{3} z-z^{4}\right)}_{\text {I, II, III, IV }} \\
& +\underbrace{\frac{3}{20} \frac{Q^{2}}{\pi^{2} \nu r^{3} h^{6}}\left(2 z^{6}-6 z^{5} h+5 z^{4} h^{2}-z h^{5}\right)}_{\text {I, III }} \\
u_{\varphi}= & \underbrace{r \frac{z}{h}}_{\text {I, II, III, IV }}+\underbrace{\frac{\rho}{60 \mu^{2}} \frac{\mathrm{d} p}{\mathrm{~d} r}\left(\frac{3 \Omega}{h} z^{5}-5 \Omega z^{4}+2 \Omega h^{3} z\right)}_{\text {IV }} .
\end{aligned}
$$

Rao I, Pahlovy III and Huang IV have to use an iteration method, to find the solutions of the velocity components, due to the fact that their simplified equations remain partial differential equations. For a comparison of the radial velocity component an integration with respect to $\varphi$ and $r$ seems suitable, which leads to the volumetric flow rate as shown earlier (Eq. (34)). The difference at this equation is a third component.

$$
Q_{r}=\int_{0}^{2 \pi} \int_{0}^{h} u_{r} r \mathrm{~d} z \mathrm{~d} \varphi=-\frac{\pi h^{3}}{6 \mu} \frac{\mathrm{d} p}{\mathrm{~d} r}+\frac{\pi r^{2} h^{3} \Omega^{2}}{20 \nu}+\frac{9 Q^{2} h}{140 \pi \nu r^{2}}
$$

The budget of these three components is shown in Figure 9 as a function of the Reynolds number $R e_{l}$, analogous to Figure 2 in the main manuscript. The third normalized term is colour-coded in blue, takes a constant value of $6.1 \times 10^{-4}$ and is thus small compared to the Poiseuille flow and the flow induced by centrifugal forces. Therefore, the simplification of neglecting this term is justified.

For the circumferential component two diagrams at the position $r=R_{1}$ for two selected angular velocities are shown in Figure 10. The Couette flow predominates both small and large angular velocities. The approximation to a linear flow profile, which consists only of the first term, accordingly, seems justified. 


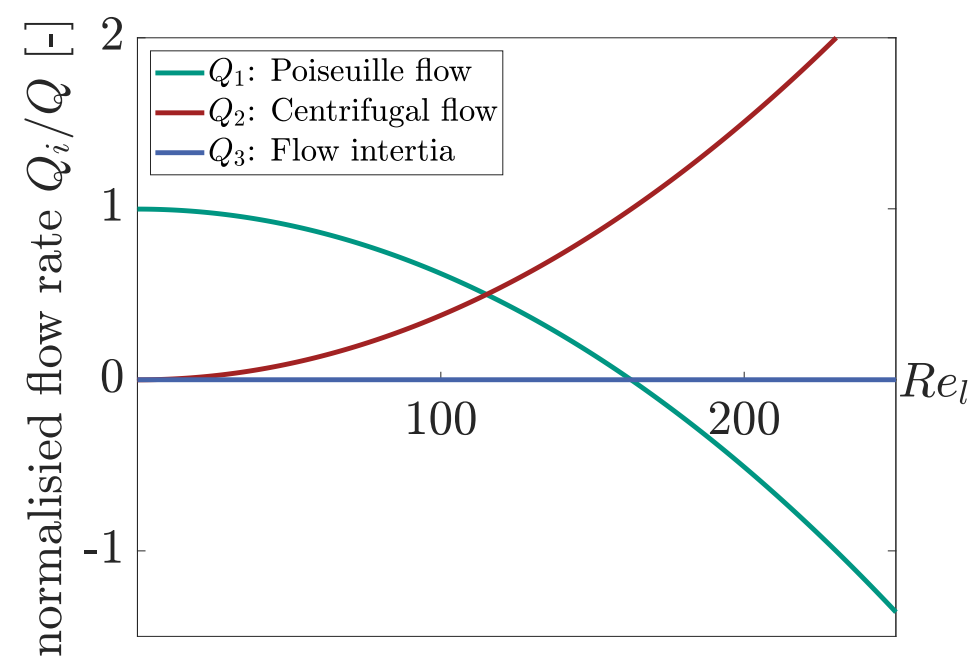

Figure 9: Budget of the volume flow rate $Q$ as a function of lubrication Reynolds number $R e_{l}$

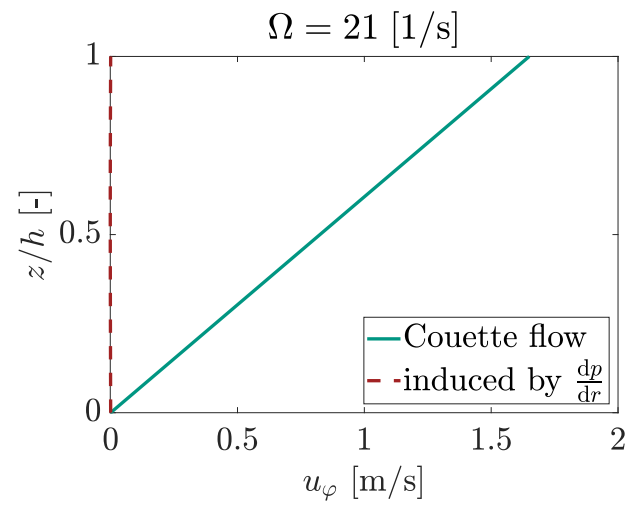

(a)

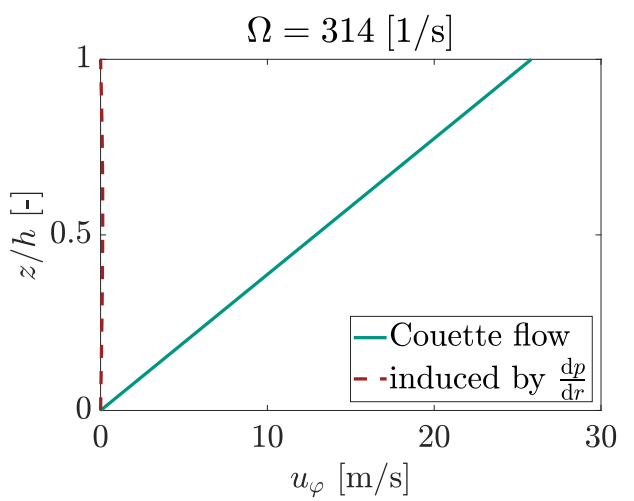

(b)

Figure 10: Budget of the circumferential velocity $u_{\varphi}$ at (a) $\Omega=211 / \mathrm{s}$ and (b) $\Omega=3141 / \mathrm{s}$ 